\title{
A numerical solution for geometrically nonlinear bending plates problems subjected to local- loads
}

\author{
Ali M. Mansour ${ }^{1, *}$, Radek Gabbasov ${ }^{2}$, and Vladimir Filatov ${ }^{3}$ \\ ${ }^{1,2,3}$ Moscow state university of civil engineering, Yaroslavskoye shosse, 26, Moscow, Russia, 129337
}

\begin{abstract}
. the following scientific content is demonstrating a new proposed numerical procedure for calculating deformations \& stresses generated on geometrically nonlinear flexible (thin) plates under local loads. solving the predominating system of differential equations is based on generalized equations of the finite difference method. the proposed algorithm is verified by an example of solving a hinged-edges plate loaded with a point load centrally.
\end{abstract}

\section{Through manuscript}

Nowadays, Flexible (thin) plates and shells are widely used in modern technology and by many various aspects such as aircraft construction, ship-building, engineering, energy, construction and much many others. The most common and universal numerical method for calculating such structures (including nonlinear calculations) is the extra-famous finite element method (FEM) [1-13]. At the same time, engineers are always in need to compare the results obtained by (FEM) (especially for performed nonlinear calculations), with the results of solutions obtained by other methods [10, 13]. Down-below, authors are suggesting a numerical technique for doing rectangular plates calculations with the aid of generalized equations of finite differences method [15]. As well-known [14], for geometrically nonlinear rectangular thin-plates, the next $4^{\text {th }}$ order differential equations system is considered to be the predominating one, which formulation can reduce to the solution to as follows:

$$
\begin{gathered}
\nabla^{2} \nabla^{2} W=\frac{H}{D}\left(\frac{\partial^{2} \Phi}{\partial y^{2}} \frac{\partial^{2} W}{\partial x^{2}}+\frac{\partial^{2} \Phi}{\partial x^{2}} \frac{\partial^{2} W}{\partial y^{2}}-2 \frac{\partial^{2} \Phi}{\partial x \partial y} \frac{\partial^{2} W}{\partial x \partial y}\right)+\frac{q}{D} \\
\nabla^{2} \nabla^{2} \Phi=E\left[\left(\frac{\partial^{2} W}{\partial x \partial y}\right)^{2}-\frac{\partial^{2} W}{\partial x^{2}} \frac{\partial^{2} W}{\partial y^{2}}\right]
\end{gathered}
$$

Where $W$ - the deflection function; $\Phi$ - the stress function; $H$ - Plate thickness; $D$ - cylindrical rigidity (stiffness).

\footnotetext{
* Corresponding author: alaa for all@hotmail.com
} 
Now, introducing dimensionless definitions for coordinates \& parameters as:

$$
\xi=\frac{x}{a} ; \eta=\frac{y}{a} ; w=\frac{W}{a} ; m=\frac{M}{q a^{2}} ; M=\frac{M_{x}+M_{y}}{1+\mu} ; p=\frac{q}{q_{0}}
$$

Where $\mu$ - Poisson's ratio; $q_{0}$ - the value of $(q)$ at any calculation node.

And, The stress function in its dimensionless form:

$$
f=\frac{\Phi}{E a^{2}}
$$

Where $E$ - modulus of elastic.

Rewriting EQ (1) with the terms of EQ (3) and EQ (4):

$$
\begin{aligned}
& \left(\frac{\partial^{2}}{\partial \xi^{2}}+\frac{\partial^{2}}{\partial \eta^{2}}\right)\left(\frac{\partial^{2} w}{\partial \xi^{2}}+\frac{\partial^{2} w}{\partial \eta^{2}}\right)= \\
& =12\left(1-\mu^{2}\right) \frac{a^{2}}{H^{2}}\left(\frac{\partial^{2} f}{\partial \eta^{2}} \frac{\partial^{2} w}{\partial \xi^{2}}+\frac{\partial^{2} f}{\partial \xi^{2}} \frac{\partial^{2} w}{\partial \eta^{2}}-2 \frac{\partial^{2} f}{\partial \xi \partial \eta} \frac{\partial^{2} w}{\partial \xi \partial \eta}\right)+\frac{a^{3}}{D} q \\
& \quad \frac{\partial^{2} w}{\partial \xi^{2}}+\frac{\partial^{2} w}{\partial \eta^{2}}=-m .
\end{aligned}
$$

Substituting with EQ (6) back into EQ (5), obtaining a bi-equational system of $2^{\text {nd }}$ order with terms of dimensionless unknowns

$$
\begin{aligned}
& \frac{\partial^{2} m}{\partial \xi^{2}}+\frac{\partial^{2} m}{\partial \eta^{2}}= \\
& =-12\left(1-\mu^{2}\right) \frac{a^{2}}{H^{2}}\left(\frac{\partial^{2} f}{\partial \eta^{2}} \frac{\partial^{2} w}{\partial \xi^{2}}+\frac{\partial^{2} f}{\partial \xi^{2}} \frac{\partial^{2} w}{\partial \eta^{2}}-2 \frac{\partial^{2} f}{\partial \xi \partial \eta} \frac{\partial^{2} w}{\partial \xi \partial \eta}\right)-\frac{a^{3}}{D} q
\end{aligned}
$$

Let the right-hand side of EQ (7) be defined as $(g)$ term:

$$
g=\frac{a^{3}}{D} q+12\left(1-\mu^{2}\right) \frac{a^{2}}{H^{2}}\left(\frac{\partial^{2} f}{\partial \eta^{2}} \frac{\partial^{2} w}{\partial \xi^{2}}+\frac{\partial^{2} f}{\partial \xi^{2}} \frac{\partial^{2} w}{\partial \eta^{2}}-2 \frac{\partial^{2} f}{\partial \xi \partial \eta} \frac{\partial^{2} w}{\partial \xi \partial \eta}\right)
$$

Then EQ (7) could be reformed as :

$$
\frac{\partial^{2} m}{\partial \xi^{2}}+\frac{\partial^{2} m}{\partial \eta^{2}}=-g
$$

From EQ (2), it's also possible to obtain a couple of $2^{\text {nd }}$ order differential equations:

$$
\begin{gathered}
\frac{\partial^{2} f}{\partial \xi^{2}}+\frac{\partial^{2} f}{\partial \eta^{2}}=-s \\
\frac{\partial^{2} s}{\partial \xi^{2}}+\frac{\partial^{2} s}{\partial \eta^{2}}=\frac{\partial^{2} w}{\partial \xi^{2}} \frac{\partial^{2} w}{\partial \eta^{2}}-\left(\frac{\partial^{2} w}{\partial \xi \partial \eta}\right)^{2}
\end{gathered}
$$


Obtaining four 2nd order differential equations of the second order: EQ (9), EQ (6), EQ (11) and EQ (10); which to be solved in terms of EQ (8), considering the right-hand side of the differential equation EQ (11).

Let the right-hand side of EQ (11) be defined as $(l)$ term $\&$ separately could be stated as:

$$
l=\frac{\partial^{2} w}{\partial \xi^{2}} \frac{\partial^{2} w}{\partial \eta^{2}}-\left(\frac{\partial^{2} w}{\partial \xi \partial \eta}\right)^{2}
$$

Then the equation takes the form:

$$
\frac{\partial^{2} s}{\partial \xi^{2}}+\frac{\partial^{2} s}{\partial \eta^{2}}=l .
$$

Solution algorithm consists of four main steps:

1. For $\left(g=\frac{a^{3}}{D} q\right) \&$ with the aid of EQ (9), the value of $(m)$ to be determined.

2. With EQ (6) \& solving the system of equations, we can find the deflection value $(w)$ at the calculated node.

3. The values of $(l),(s)$ and then $(f)$ to be determined using EQ (12) \& EQ (13) and EQ (10) respectively.

4. The same wise, the new value of $(g)$ to be calculated using EQ (8).

The previous steps to be repeated while moving to a new calculation cycle (new calculation node), considering that

$$
g=\frac{1}{2}\left(g_{n-1}+g_{n}\right)
$$

where $g_{n}$ - the value of $(g)$ out of the last calculation cycle.

$g_{n-1}$ - the value of $(g)$ out of the penultimate (before-last) calculation cycle.

The generalized equations of the finite difference method [15] are used to solve the above differential equations, which consider the discontinuity of the unknown function and its first derivative represented by the right-hand sides of the previous differential equations.

For the aim of illustrating the presented algorithm, Calculation of local load case is considered; Suppose that a load in the form of two symmetrically triangles acts on the center of a 4-sided hinge-supported square thin-plate as shown in (Fig. 1), noting that the load takes that triangular form affecting a small loaded area, thus; we can say that it acts as a point load relatively governed by the following formula:

$$
P=4 \frac{1}{2} k h_{a} q_{0},
$$


Where $q_{0}$ - load intensity; $h$ - meshing step; $a$ - square plate side length; $K$ - coefficient that represents the contribution of the loaded part $(h)$.

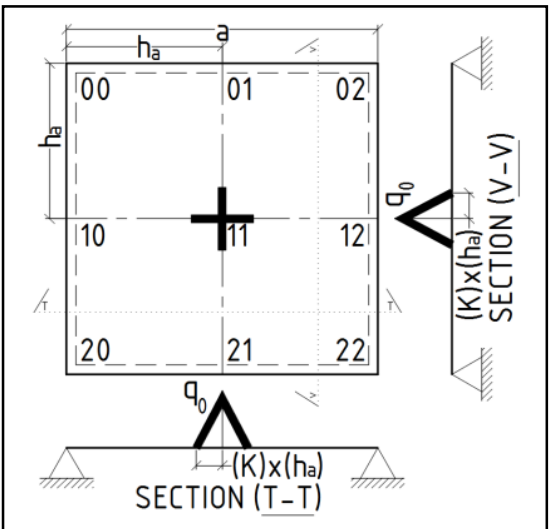

Fig. 1. A 4-sided hinge-supported square thin-plate loaded centrally.

Simplifying EQ (14), separating $\left(q_{0}\right)$ in a formula individual side getting that $q_{0}=\frac{P}{2 k h a}$ which implies the load intensity in its dimensionless form $\left(q_{0}^{*}\right)$ as:

$$
q_{0}^{*}=\frac{q_{0}}{P / a}=\frac{1}{2 k h}
$$

In the verifying example currently studied here, and in order to go through the solution steps; it is necessary to the considered the approximation of the differential EQ (9) taking into account the boundary conditions and the stripped pattern of the load centered onto the plate.

As per (3.1.24) (Equation No.24, $1^{\text {st }}$ Clause at the $3^{\text {rd }}$ chapter) reference [15] multiplied by $\left(h^{2}\right)$, applied to node 11 (Fig. 1):

$$
4 m_{01}-4 m_{11}+h\left(\Delta^{\mathrm{I}-\mathrm{II}} m_{11}^{\xi}+\Delta^{I-I I I} m_{11}^{\eta}\right)=-h^{2} g_{11}
$$

Where the shear force discontinuities are involved into calculations according to EQ

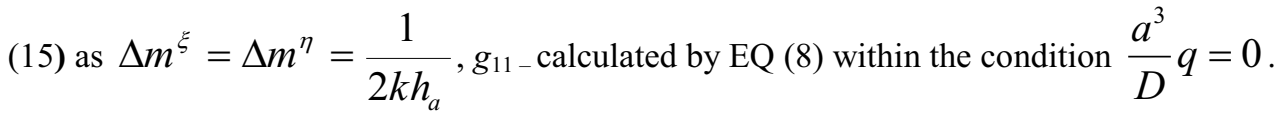

As well as boundary conditions know cause the bending moments to be always equal to zero on its nodes, thus $m_{0 j}=0$; using EQ (16) we get:

$$
m_{11}=\frac{h^{2}}{4} g_{11}+\frac{h}{4} \frac{1}{2 k h} 2 \text {. }
$$

Concerning the non-linear formulation of our problem, besides $q=0-g_{11}=0$, EQ (17) generates;

$$
m_{11}=\frac{1}{4 k} \text {. }
$$

When $(k \rightarrow 0) ;\left(m_{11} \rightarrow \infty\right)$ 
For deflections determination, the difference syntax of EQ (6) should be applied to node 11. Once more using (3.1.25) of reference [15], multiplied by $\left(h^{4}\right)$ getting:

$$
4 w_{01}-4 w_{11}=-h^{2} m_{11} \text {. }
$$

From the boundary conditions $\rightarrow w_{01}=0$, then for $h=1 / 2, g_{11}=0$ and the value of $m_{11}$ (calculated before via EQ (17)), EQ (19) gives us:

$$
w_{11}=\frac{h^{2}}{4} m_{11}=\frac{h^{2}}{4} \frac{1}{4 k}=\frac{1}{4} \frac{1}{2^{2}} \frac{1}{4} \frac{1}{k}=\frac{1}{64 k} .
$$

In accordance to the solution algorithm stated above, difference syntax of EQ (9) to be written taking into calculations $g_{11}=0$, the algorithm is validated through EQ (18) that originally came from EQ (16). From EQ (6), associated with the resulted value of $m_{11}$; we can find the value of $w_{11}$. Also, with the aid of EQ (12); we can get the value of $(l)$, the same fashion, solving EQ (13) generates the value of $(s)$ noting that all these $2^{\text {nd }}$ order differential operators are to be written in their difference forms.

$$
\text { On that, when }\left(g_{11}=0\right) \rightarrow m_{11}=\frac{1}{4 k} ; w_{11}=\frac{1}{64 k} .
$$

Returning to EQ (12) for expressing $l$ in terms of $w_{11}: l_{11}=64 w_{11}^{2}$ and to EQ (13) for determining the value of $s$ at node 11. Next to that; EQ (10) allows us to find the value of $\left(f_{11}\right)$. By the end of this calculation cycle, confirming the value of $g_{11}$ (on bases of EQ (8)) when $q=0$ as follows:

$$
g_{11}=96\left(1-\mu^{2}\right) \frac{a^{2}}{H^{2}} \frac{1}{h^{4}} f_{11} w_{11} .
$$

Finally, the previous cycle is repeated. Exact data for solving the problem numerically could be taken from $\left(84^{\text {th }}\right.$ page $)$ reference $[\mathbf{1 4}]$.

Progress of solution to be monitored in the light of the proceeded algorithm. For $g_{11}=0$ and depending on EQ (17); an initial value of $m_{11}$ to be determined, and once more substituting in EQ (17) when $k=1$; getting that $\left(m_{11}=0.25\right)$. difference approximation for the differential equation EQ (6) gives us:

$$
w_{11}=\frac{1}{4} \frac{1}{2^{2}} m_{11} \text {. }
$$

Further proceeding through the previous mentioned algorithm results in the values; $s_{11}=-4 w_{11}^{2} \& f_{11}=0,0625 s_{11}$ and those values forming finally:

$$
52,73 m_{11}^{3}+m_{11}=0,25 \text {. }
$$

At the end \& after determining the value of $m_{11}=0,132$, following the same technique enables us to calculate the other parameters with the aid of the previous set of equations. Now, it became possible to solve that kind of problems, such that in reference [14], relatively;

$$
\varphi=\frac{W}{H}
$$

Where $W$ - the maximum deflection; $H$ - Plate thickness

The numerical solution presented in this paper and other more similar numerical proposals become more acceptable in practical calculations, since all such solutions find their way to be performed on modern computers... 


\section{References}

1. T. Belytscko, W. Liu, B. Moran, Nonlinear finite elements for continua and structures. JWiley \& Sons, 300 (2000)

2. J. Bonet, R. Wood, Nonlinear continuum mechanics for finite element analysis.Cambridge University Press, 248 (1997)

3. M.A. Crisfield, Non-linear finite elements analysis of solids and structures. J Wiley \& Sons, 1, 362 (1997)

4. P. Kyther, D. Wie, An introduction to linear and nonlinear finite element analysis. Birkhauer Verlag, 445 (2004)

5. J.N. Reddy, An introduction to nonlinear finite element analysis. Oxford University Press, 488 (2004)

6. A.N. Danilin, N.N. Zuev, D.V. Snegovsky, V.I. Shalashilin, CAD and graphics. 4, 26$31(2000)$

7. V.V. Galishnikova, International Journal for Computational Civil and Structural Engineering. 5, 35-44 (2009)

8. V.V. Galishnikova, Construction mechanics of engineering structures and structures, $\mathbf{1}$, 42-50 (2010)

9. V.D. Kurguzov, Izvestiya Vysshikh Uchebnykh Zavedenii. Building. 3-4, 14-22 (2009)

10. V.D. Kurguzov V.D. Computational technologies. 14, 70-80 (2009)

11. S.V. Levyakov Applied Mechanics and Technical Physics. 53, 128-136 (2012)

12. A.O. Scherbakova, Vestnik SUSU. Series Mathematics. Mechanics. Physics. 32, 83-91 (2011)

13. V.A. Ignatiev, A. V. Ignatie, E.V.Onischenko, Vestnik MGSU. 2, 20-33 (2016)

14. A.S. Volmir Flexible plates and shells. Gos. Ed. Technical and Theoretical Literature, Moscow, 419 (1956)

15. R.F. Gabbasov, A.R. Gabbasov, V.V. Filatov Numerical construction of discontinuous solutions to the problems of structural mechanics. Ed. ASV, Moscow, 280 (2008) 